\title{
MÉTODO DE CALIFICACIÓN DE ATRIBUTOS DE CALIDAD EN PRODUCTOS DE SOFTWARE MÉDICO REGULADO USANDO PROCESO ANALÍTICO JERÁRQUICO Y RÚBRICA DE EVALUACIÓN
}

QUALIFICATION METHOD OF QUALITY ATTRIBUTES IN MEDICAL SOFTWARE PRODUCTS REGULATED BY THE HIERARCHICAL ANALYTICAL PROCESS AND THE EVALUATION RUBRIC

Jonathan Venegas-Sandoval ${ }^{1}$

Jonathan.venegas.sandoval@gmail.com

Francisco J. Álvarez-Rodríguez ${ }^{2}$

fjalvar.uaa@gmail.com

1 Universidad Juárez Autónoma de Tabasco

Avenida Universidad S / N Villahermosa, Tabasco, México

${ }^{2}$ Avenida Universidad \# 940

Ciudad Universitaria, Aguascalientes, Ags, México 


\title{
RESUMEN
}

Las aplicaciones móviles que diagnostiquen enfermedades o funcionen como equipo médico pueden poner en peligro la salud de las personas cuando no funcionan adecuadamente. El control sobre la calidad de las aplicaciones en Google Play se basa en la usabilidad a partir de calificaciones emitidas por los usuarios. En App Store, los controles para las aplicaciones son más amplios, sin embargo, no validan por parte de expertos las aplicaciones que realizan funciones de dispositivos médico. En el presente documento se muestra una propuesta de calificación de aplicaciones móviles para el área médica utilizando una lista de atributos de calidad extraídos de normativa regulatoria. El método presentado consiste en complementar el Proceso Analítico Jerárquico (AHP por sus siglas en inglés) con una rúbrica de evaluación para determinar un grupo de aplicaciones móviles médicas que cumpla con una lista de atributos de calidad. Como caso experimental se evaluaron cinco aplicaciones móviles médicas disponibles en Google Play que miden el ritmo cardíaco con alta tasa de descargas, la menos descargada con 10,600 y una calificación de 4,5 estrellas (en un rango de 0 a 5 estrellas máximas posible), la más descargada con 198,633 descargas y una calificación de 4 estrellas.

\section{PALABRAS CLAVE}

Calidad de Software, Proceso Analítico Jerárquico, Calificación de Software

\begin{abstract}
Mobile applications that diagnose diseases or devices such as medical equipment can endanger people's health when they have no problems. The control over the quality of applications in Google Play is based on usability according to the ratings issued by users. In the App Store, the controls for the applications are broader, however, they do not validate by experts the applications that operate functions of medical devices. This document shows a proposal for the qualification of mobile applications for the medical area using a list of quality attributes extracted from regulatory regulations. The method presented consists of complementing the hierarchical analytical process (AHP) with an evaluation publication to determine a group of mobile medical applications that includes a list of quality attributes. As an experimental case, five mobile applications available on Google Play were evaluated, which measured heart rate with a high download speed, the lowest downloaded with 10,600 and a 4,5 star rating (in a range of 0 to 5 possible maximum stars), the most downloaded with 198,633 downloads and a 4 star rating.
\end{abstract}

\section{KEYWORDS}

Software Quality, Hierarchical Analytical Process, Software Qualification 


\section{INTRODUCCIÓN}

Los productos de software móvil médico deben ser analizados para determinar confiabilidad y calidad debido a los riesgos potenciales para los usuarios. Instituciones e investigadores verifican y validan de forma aleatoria este tipo de aplicaciones móviles encontrando problemas de confiabilidad, seguridad y control en estos productos:

"En algo más de 1 de cada 5, sus responsables afirmaban que podían curar trastornos médicos... Cerca del $43 \%$ usaban dispositivos del propio terminal o sonidos como medios para tratamientos, sin ser dispositivos médicos" (García y Martínez, 2016, p. 30)

El método usado principalmente para determinar la calidad de las aplicaciones de salud se enfoca en analizar los productos ofertados y analizar los niveles de calidad y el posible peligro que puede representar para la salud (Fernandez y Ramos, 2014, p.100). Es el caso de MYHEALTHAPPS desarrollada por Patient View un portal británico que valida y verifica aplicaciones móviles médicas de todo el mundo basándose en las experiencias de uso y clasificándolas por tipo e idioma entre otras, sin embargo:

"La mayoría de las Apps de salud carecen de información sobre su autoría.... Los resultados de diversos estudios han revelado que menos del 35\% de las Apps de salud tienen la participación de expertos médicos durante su desarrollo y que el

$86 \%$ de las Apps revisadas sobre control del dolor no tenían participación sanitaria profesional en su desarrollo" (García y Martínez, 2016, p. 30)

Cuando existe riesgo para la salud de la población las instituciones gubernamentales regulan y controlan productos o mercados, en Colombia la división de gobierno encargada de esta labor es el Instituto Nacional de Vigilancia de Medicamentos y Alimentos (INVIMA) la cual como su nombre indica regula los medicamentos, alimentos y productos que puedan poner en riesgo la salud de las personas, para el caso de tecnología regula los dispositivos médicos pero no el software médico o las aplicaciones móviles que convierten un teléfono inteligente en un dispositivo médico (ISO 13485, 2016 p.18). En México se encuentra la Comisión Federal para la Protección contra Riesgos Sanitarios (COFEPRIS) la cual otorga licencias para el uso, transporte, almacenamiento y venta de productos regulados. Cuando una persona desea vender un producto en una farmacia debe tener una licencia de la COFEPRIS para verificar que el almacenamiento, manipulación y condiciones en las que vende los productos regulados no atentan contra la salud. Teniendo en cuenta que la oferta de aplicaciones para el ámbito médico aumenta constantemente (García y Martínez, 2016, pp. 29-30) y que el funcionamiento incorrecto de una aplicación médica puede generar una afectación de salud, alguna división gubernamental mexicana debería controlar el mercado de las Apps de salud. En otros países algunas instituciones como la agencia de administración de drogas y alimentos de los Estados Unidos FDA (por sus siglas 
en inglés) se han adaptado a estos nuevos mercados para regular las aplicaciones móviles que puedan poner en peligro la vida de seres humanos. Esta agencia tiene amplia reputación y experiencia en la protección de la salud humana regulando drogas, alimentos y para este caso en particular, aplicaciones de software médico, la experiencia que posee regulando medicamentos, alimentos y dispositivos médicos la trasladó a las aplicaciones móviles, lo que garantiza que el control es exhaustivo y eficiente debido a su reputación, pero la agencia no enfoca sus esfuerzos en todas las aplicaciones móviles médicas. En España la Agencia de Calidad Sanitaria de Andalucía presenta la Estrategia de calidad y seguridad en aplicaciones móviles de salud, con el objetivo de brindar una serie de recomendaciones para desarrollar aplicaciones médicas de cualquier tipo, garantizando calidad y pertinencia en los productos. Esta agencia brinda un distintivo que identifica a la aplicación como una aplicación de calidad. Por ello se busca que las aplicaciones móviles médicas sean seguras y confiables, que estén en condiciones de realizar la actividad por la cual fueron desarrolladas (García y Martínez, 2016, p. 32). Sin embargo, no existe una herramienta para calificar Apps de la salud, la elección de software móvil por parte de un usuario potencial se basa en la calificación de otros usuarios a partir de la usabilidad. Sin embargo, dicha calificación no es técnica, se basa en la percepción de éxito o afinidad con el diseño y estructura de la aplicación móvil, lo que da una idea errada de calidad en la aplicación. Si a lo anterior se agrega que la usabilidad tiene diferente interpretación en otras ramas de la ciencia (Muñoz, Piattini, y Moraga, 2010 p. 62), resulta poco claro el termino para determinar la calidad de una app.

Para poder confiar la salud de un usuario a una aplicación de salud es necesaria una calificación más allá de la usabilidad, se necesita una calificación técnica avalada por expertos del área que siga una lista de atributos definida (Redrován, Loja, Correa y Piña 2017 pp. 94-113). Este método beneficia a pacientes, familiares y personal médico; sin embargo, falta establecer bajo qué condiciones se realizará y quién realizará la calificación de las aplicaciones. Esta propuesta presenta una herramienta que podría ser un complemento a las normas de calidad de productos de software o una herramienta propuesta para calificar aplicaciones en las diferentes plataformas de distribución digital. La propuesta para calificar aplicaciones móviles médicas según el nivel de calidad se basa en una lista de atributos que toda aplicación móvil debe tener según las normas ISO de calidad de software (Muñoz, Piattini, y Moraga, 2010 p. 92), donde un atributo es una característica de calidad que permite a los usuarios aceptar un producto (Fernández y Ramos, 2014 p. 99) y a su vez determina cuando una aplicación móvil puede ser utilizada sin poner en riesgo la salud del usuario.

Este trabajo se dividió en tres secciones, la primera consistió en determinar elementos considerados como críticos para la calidad de un producto de software móvil en el área médica, tomando como fuente a las instituciones certificadoras y reguladoras, incluyendo, teoría básica de validación y verificación de sistemas computarizados. Sin embargo, a pesar de que una gestión efectiva de tecnología y del proceso de desarrollo impacta en la calidad de los productos de software (Tuya, 
Ramos, y Dolado, 2007 p.69), las instituciones reguladoras fijan sus objetivos en la documentación y en el producto, más que en el modelo de proceso o de gestión utilizado, por ello, estos aspectos están fuera del alcance del presente estudio. El segundo paso consistió en utilizar la herramienta AHP diseñada por Thomas L. Saaty en la década de los noventa para calificar aplicaciones móviles. Complementando dicho método se propone una rúbrica de evaluación como métrica para calificar las aplicaciones y determinar cuándo pueden ser recomendadas al usuario utilizando el rango de evaluación del AHP más el número cero. Finalmente, se muestra una prueba de aplicación realizada a cinco aplicaciones móviles concernientes al monitoreo cardíaco.

\section{MÉTODO}

Las herramientas de selección multicriterio son utilizadas ampliamente en industria para evaluar proveedores, clientes o proyectos, el Proceso Analítico Jerárquico tiene la posibilidad de ser usado en diferentes núcleos de la ciencia sin afectar los resultados (Saaty, 2011, p. 101). Sin embargo, antes de usar esta herramienta se presenta un grupo de herramientas de selección multicriterio.

\begin{tabular}{|c|c|c|}
\hline $\begin{array}{l}\text { Herramienta } \\
\text { multicriterio }\end{array}$ & Similitudes con AHP & Diferencias con AHP \\
\hline El método Macbeth & $\begin{array}{l}\text { Se basa en expertos, } \\
\text { evalúa cualitativo y } \\
\text { cuantitativo }\end{array}$ & $\begin{array}{l}\text { Atributos o características } \\
\text { Finitas }\end{array}$ \\
\hline $\begin{array}{l}\text { Teoría de la utilidad } \\
\text { Multiatributo }\end{array}$ & $\begin{array}{ll}\text { Tiene } & \text { alternativas } \\
\text { características y criterios } \\
\text { como partes de la } \\
\text { herramienta. }\end{array}$ & $\begin{array}{l}\text { Se requiere de alto } \\
\text { conocimiento por parte del } \\
\text { decisor }\end{array}$ \\
\hline $\begin{array}{l}\text { Relaciones } \\
\text { Superación }\end{array}$ & $\begin{array}{l}\text { Número finito de criterios } \\
\text { para hacer comparación } \\
\text { uno por uno. }\end{array}$ & $\begin{array}{lrr}\text { Usan } & \text { como mecanismo } \\
\text { básico } & \text { comparaciones } \\
\text { binarias } & \text { de alternativas }\end{array}$ \\
\hline $\begin{array}{l}\text { Proceso analítico } \\
\text { en red }\end{array}$ & $\begin{array}{l}\text { Misma base teórica del } \\
\text { AHP }\end{array}$ & $\begin{array}{l}\text { Permiten relaciones de } \\
\text { interdependencia entre } \\
\text { elementos de distintas ramas }\end{array}$ \\
\hline Ponderación lineal & $\begin{array}{l}\text { Sencillo de utilizar. Aplica } \\
\text { pesos a los criterios. }\end{array}$ & $\begin{array}{l}\text { Se emplea cuando se dispone } \\
\text { de poca información, en } \\
\text { situaciones de incertidumbre }\end{array}$ \\
\hline
\end{tabular}

Tabla 1. Similitudes y Diferencias de AHP con otras Herramientas de Análisis Multicriterio (Venegas, 2019 p. 26)

La ventaja de esta herramienta radica en la posibilidad de evaluar "k" alternativas (aplicaciones móviles) y " $n$ " criterios (atributos de calidad). Es posible cuantificar características o criterios tanto cualitativos como cuantitativos, permitiendo una elección neutral de la mejor opción (App móvil médica) para el usuario. El objetivo de la herramienta AHP es elegir la mejor alternativa entre múltiples opciones utilizando como elemento de decisión la comparación por pares. Esta herramienta 
se compone de tres niveles; el objetivo, los criterios o características y las alternativas. Las alternativas son las opciones disponibles para elegir, las características son elementos base para tomar la decisión y el objetivo es elegir la alternativa óptima. El proceso utiliza la comparación por pares a partir de una escala de calificación por preferencias donde; $1=$ igualmente preferente, $3=$ moderadamente preferente, $5=$ fuertemente preferente, $7=$ muy fuertemente preferente, 9 = extremadamente preferente.

Fig. 1: AHP aplicado a atributos de calidad de aplicación móvil.

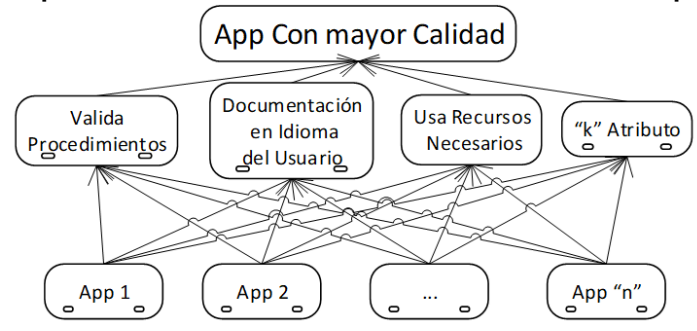

Las calificaciones por pares se realizarán para comparar las características con ellas mismas, posteriormente se comparan las alternativas con sí mismas bajo el enfoque de cada característica.

\subsection{Aportación}

El Proceso Analítico Jerárquico es una buena herramienta para encontrar la mejor alternativa de un grupo de opciones aun cuando todas las alternativas son malas. Por ejemplo, supóngase que se presentan 4 aplicaciones como alternativas y ninguna cuenta con características de calidad aceptables para la rama médica, la herramienta permite seleccionar la mejor propuesta sin importar si todas las propuestas tienen bajo nivel de calidad o son "malas" lo que significa que utilizando el método de selección multicriterio por sí solo es insuficiente para determinar cuál aplicación es recomendable utilizar.

Fig. 2: AHP con Rúbrica de evaluación
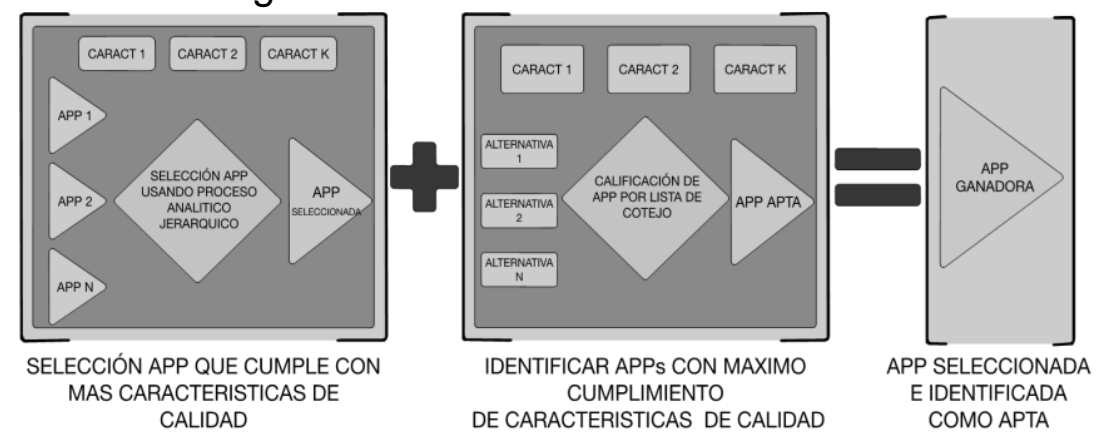

Por esta razón se propone una rúbrica de evaluación utilizando la escala de calificación presentada por Thomas Saaty para el AHP, agregando el número cero y contando con 3, 5, 7 y 9 como únicos rangos de calificación. Posterior a la calificación de cada aplicación usando como guía la rúbrica de evaluación en la 
Tabla 6, se propone una condición de conteo de valores para validar el máximo cumplimiento de características de calidad. Esta propuesta de métrica no puede determinar la mejor App de forma individual, puesto que cuando un grupo de aplicaciones cumple las mismas condiciones de conteo será calificado con el mismo valor, siendo difícil calificar el grado de calidad de las aplicaciones. La lógica de trabajo se divide en dos partes (ver Figura 2), donde una parte es la selección de la mejor alternativa utilizando el AHP y la otra se encarga de evaluar por separado cada aplicación para validar el cumplimiento de los atributos críticos de calidad. La App móvil óptima se encuentra cuando la aplicación con el puntaje más alto en el Proceso Analítico Jerárquico se encuentra en el rango de aplicaciones aceptadas en la métrica de lista de cotejo. Cuando ninguna App obtiene la calificación adecuada en el sistema de calificación por lista de cotejo, la técnica de decisión multicriterio estará entregando la calificación de las aplicaciones teniendo en cuenta que ninguna es apta para su uso, es decir, el AHP determinaría la mejor aplicación entre un grupo de aplicaciones con calidad no aceptable.

\section{CASO DE APLICACIÓN: CATEGORIZACIÓN DE CINCO APLICACIONES MÓVILES MÉDICAS QUE MIDEN RÍTMO CARDÍACO}

Para el caso ejemplo se determinaron cinco aplicaciones móviles que miden el ritmo cardíaco. A pesar de tener un funcionamiento similar (todas solicitan poner el dedo índice en la cámara del dispositivo móvil) algunas miden nivel de oxígeno en la sangre e incluso el estado de ánimo. Pero el objetivo principal de las aplicaciones seleccionadas es la determinación del ritmo cardíaco, dichas mediciones se pueden usar de forma general para actividades recreativas, deportivas o para implicaciones médicas, en cualquiera de los casos podría comprometer la salud del usuario y en caso de ser usada para fines médicos la aplicación funcionaría en un equipo móvil como un dispositivo médico. Para calificar de mayor a menor las cinco aplicaciones y determinar cuál de ellas se puede recomendar al usuario para su uso se siguieron dos pasos:

Paso 1 Determinación de elementos de calidad críticos para una aplicación móvil del área médica: Las características de calidad de software propuestas por los diferentes organismos basan sus propuestas en la norma ISO/IEC 9126 desarrollada para identificar los atributos de calidad para un software y de las cuales se extraen los principales atributos de calidad y en la ISO/IEC 14598 la cual da una descripción de los procesos para evaluar software y guías para evaluar la calidad de los productos. Según los atributos de calidad presentes en la ISO/IEC 9126 para los productos software de la cual se basan las agencias reguladoras para hacer recomendaciones de calidad para aplicaciones móviles para la salud se utiliza una muestra de atributos para considerar una aplicación médica móvil como confiable. Se debe aclarar que la propuesta puede evaluar " $k$ " características de calidad, sin embargo, se presentan nueve características a modo de muestra para poder probar la herramienta evaluadora. 


\begin{tabular}{|c|c|c|}
\hline & Elementos de calidad Críticos & ¿Quién propone? \\
\hline $\mathrm{a}$ & $\begin{array}{l}\text { Se validan todos los procedimientos } \\
\text { de registros electrónicos y firmas } \\
\text { electrónicas. }\end{array}$ & $\begin{array}{l}\text { Titulo } 21 \text { del código de regulaciones } \\
\text { federales de los Estados Unidos, } \\
\text { Parte } 11 \text { del } 21 \text { CFR }(11.10-11.30 \text { - } \\
11.50-11.200-11.300)\end{array}$ \\
\hline$b$ & $\begin{array}{l}\text { cumentación completamente en } \\
\text { ma del usuario comprensible y } \\
\text { iciente sobre uso de la aplicación, } \\
\text { ntes de información y } \\
\text { icionamiento de la aplicación. }\end{array}$ & $\begin{array}{l}\text { Propuesto a partir del análisis de } \\
\text { características críticas del presente } \\
\text { artículo (Paso } 1 \text { de esta sección) }\end{array}$ \\
\hline$c$ & $\begin{array}{l}\text { iza el dispositivo móvil como un } \\
\text { upo médico regulado (Mide, } \\
\text { nitorea y/o proporciona datos de } \\
\text { ud) }\end{array}$ & $\begin{array}{l}\text { Documento número } 1741 \text { de la } \\
\text { Agencia de drogas y alimentos de los } \\
\text { Estados Unidos (FDA) apéndice C }\end{array}$ \\
\hline$d$ & $\begin{array}{l}\text { licita y utiliza únicamente los } \\
\text { ursos necesarios para cumplir con } \\
\text { bjetivo del diseño del producto. }\end{array}$ & $\begin{array}{l}\text { Propuesto a partir del análisis de } \\
\text { características criticas }\end{array}$ \\
\hline$e$ & $\begin{array}{l}\text { eador } \\
\text { es del } \\
\text { ntacto }\end{array}$ & $\begin{array}{l}\text { Agencia de Calidad Sanitaria de } \\
\text { Andalucía }\end{array}$ \\
\hline f & $\begin{array}{l}\text { "La App de salud advierte de aquellas } \\
\text { actualizaciones que inciden } \\
\text { modifican funcionamientos } \\
\text { contenidos sobre salud o cualquier } \\
\text { otro dato sensible." }\end{array}$ & $\begin{array}{l}\text { Agencia de Calidad Sanitaria de } \\
\text { Andalucía }\end{array}$ \\
\hline$g$ & $\begin{array}{l}\text { "Se identifican los riesgos } \\
\text { manejo de la App de salu } \\
\text { suponer para la seguric } \\
\text { paciente." y toma las } \\
\text { preventivas respectivas. }\end{array}$ & $\begin{array}{l}\text { Agencia de Calidad } \\
\text { Andalucía }\end{array}$ \\
\hline & $\begin{array}{l}\text { salud contiene la fecha de } \\
\text { visión realizada sobre el } \\
\text { licado." }\end{array}$ & $\begin{array}{l}\text { Agencia de Calidad Sanitaria de } \\
\text { Andalucía }\end{array}$ \\
\hline & $\begin{array}{l}\text { "La App de salud dispone de } \\
\text { sistema de ayuda sobre su mane } \\
\text { amplio y suficiente por medio escr }\end{array}$ & $\begin{array}{l}\text { Agencia de Calidad Sanitaria } \\
\text { Andalucía }\end{array}$ \\
\hline
\end{tabular}

Tabla 2. Elementos de Calidad para Aplicaciones Móviles (FDA, 2016)

Paso 2 Uso de rúbrica de evaluación y herramienta proceso analítico jerárquico para clasificar aplicaciones médicas: La propuesta de evaluación de aplicaciones móviles médicas se realizará en dos etapas: en la primera se utiliza el Proceso Analítico Jerárquico para calificar las aplicaciones móviles médicas. En la segunda se evaluará por medio de la lista de cotejo los atributos de calidad presentes en la aplicación, como resultado se genera un dictamen de calificación. Al finalizar las 
dos etapas se obtendrán; la aplicación que más cubre las características establecidas según los expertos y las aplicaciones recomendadas por su calidad funcional.

\subsection{Aplicando Proceso Analítico Jerárquico}

El objetivo es seleccionar entre un grupo de opciones la aplicación móvil que cumpla de mejor manera los atributos de calidad. El primer paso de la metodología consiste en hacer una evaluación por pares de los nueve atributos de calidad. En la Tabla 3 se evalúa la importancia de cada característica con las demás, siendo la calificación 1 en la diagonal principal debido a que se compara una característica con ella misma (Escala de preferencias establecida por Thomas Saaty). En el procedimiento una vez se tiene la comparación por pares por parte de los expertos Tabla 3 se suman las columnas y se divide cada elemento por el total de cada columna los resultados.

\begin{tabular}{|c|c|c|c|c|c|c|c|c|c|}
\hline \multicolumn{10}{|c|}{ Características de Calidad } \\
\hline Características & $\mathbf{a}$ & $\mathbf{b}$ & $\mathbf{c}$ & $\mathbf{d}$ & $\mathbf{e}$ & $\mathbf{f}$ & $\mathbf{g}$ & $\mathbf{h}$ & $\mathbf{i}$ \\
\hline $\mathrm{a}$ & 1 & 9 & 1 & 9 & 9 & 9 & 7 & 7 & 5 \\
\hline $\mathrm{b}$ & $1 / 9$ & 1 & $1 / 9$ & 3 & 3 & 5 & 3 & 3 & 5 \\
\hline $\mathrm{c}$ & 1 & 9 & 1 & 9 & 9 & 5 & 5 & 9 & 7 \\
\hline $\mathrm{d}$ & $1 / 9$ & $1 / 3$ & $1 / 9$ & 1 & 7 & 3 & 3 & 9 & 5 \\
\hline $\mathrm{e}$ & $1 / 9$ & $1 / 3$ & $1 / 9$ & $1 / 7$ & 1 & 3 & 5 & 9 & 7 \\
\hline $\mathrm{f}$ & $1 / 9$ & $1 / 5$ & $1 / 5$ & $1 / 3$ & $1 / 3$ & 1 & 3 & 9 & 9 \\
\hline $\mathrm{g}$ & $1 / 7$ & $1 / 3$ & $1 / 5$ & $1 / 3$ & $1 / 5$ & $1 / 3$ & 1 & 9 & 9 \\
\hline $\mathrm{h}$ & $1 / 7$ & $1 / 3$ & $1 / 9$ & $1 / 9$ & $1 / 9$ & $1 / 9$ & $1 / 9$ & 1 & 3 \\
\hline $\mathrm{i}$ & $1 / 5$ & $1 / 5$ & $1 / 7$ & $1 / 5$ & $1 / 7$ & $1 / 9$ & $1 / 9$ & $1 / 3$ & 1 \\
\hline
\end{tabular}

Tabla 3. Comparación por Pares de Características

En la Tabla 5 se observa que las características más importantes son la a) con un $29 \%$ y la característica c) con un $27,45 \%$, por otra parte, la aplicación menos importante según la opinión de los expertos es la i) con una calificación de $2 \%$. Las características fueron evaluadas, pero es necesario corroborar que la tasa de consistencia (CR por sus siglas en ingles) de la matriz no supere el $10 \%$. Esto quiere decir, que las evaluaciones de los expertos para la matriz inicial de la Tabla 3 son coherentes. Para poder calcular la tasa de consistencia CR en Figura 3 es necesario encontrar el índice de consistencia el cual se representa con $\mathrm{Cl}$, por sus siglas en inglés (Saaty, 2011, p180) 
Fig. 3: Calculo de Tasa de Consistencia o CR

$$
\begin{aligned}
& C I=(\lambda \max -n) /(n-1) \\
& C R=C I / R I<0,1 \sim 10 \% \\
& C R=13,78074 / 1,45=9,5039
\end{aligned}
$$

El cálculo de $\lambda \max$ corresponde a la multiplicación de los valores resultado por el total de la suma de cada columna. Es necesario utilizar también una tabla de índices de consistencia aleatoria o RI que se muestra en la Tabla 4.

\begin{tabular}{|c|c|c|c|c|c|c|c|c|c|c|}
\hline $\mathrm{N}$ & 1 & 2 & 3 & 4 & 5 & 6 & 7 & 8 & 9 & 10 \\
\hline $\mathrm{Rl}$ & 0 & 0 & 0,58 & 0,9 & 1.12 & 1,24 & 1,32 & 1,41 & 1,45 & 1,49 \\
\hline
\end{tabular}

Tabla 4. Índices de Consistencia Aleatoria

La tasa de consistencia CR es de 9,50\% (ver Figura 3), por lo cual se puede considerar que la matriz inicial aportada por los expertos al evaluar las nueve características es consistente, validando los resultados de esta primera etapa. EI siguiente paso es evaluar por pares a las alternativas respecto de cada

\begin{tabular}{|c|c|c|c|c|c|c|c|c|c|c|}
\hline \multicolumn{11}{|c|}{ Tabla de Resultados (Calificación de App Médicas) } \\
\hline APP & $\begin{array}{c}a \\
0.2\end{array}$ & \begin{tabular}{|c|}
$b$ \\
0.1
\end{tabular} & $\begin{array}{c}\text { C } \\
0.27\end{array}$ & \begin{tabular}{|c|}
$d$ \\
0.09
\end{tabular} & \begin{tabular}{|c|}
$e$ \\
0.08 \\
\end{tabular} & \begin{tabular}{|c|}
$f$ \\
0.07
\end{tabular} & \begin{tabular}{|c|}
$g$ \\
0.06
\end{tabular} & $\begin{array}{c}\mathrm{h} \\
0.02\end{array}$ & \begin{tabular}{|c|}
$\mathrm{i}$ \\
0.02
\end{tabular} & Calificación \\
\hline $\begin{array}{c}\text { App } \\
1\end{array}$ & 20 & 3,60 & 20 & 4,36 & 7,32 & 20 & 16,5 & 6,31 & 7,69 & 15,3 \\
\hline $\begin{array}{c}\text { App } \\
2\end{array}$ & 20 & 11,4 & 20 & 46,4 & 56,5 & 20 & 16,5 & 44,2 & 23,08 & 25,1 \\
\hline App & 20 & 28,3 & 20 & 8,88 & 6,99 & 20 & 6,31 & 16,5 & 23,08 & 17,8 \\
\hline $\begin{array}{c}\text { App } \\
4\end{array}$ & 20 & 28,3 & 20 & 20,1 & 18,6 & 20 & 44,1 & 16,5 & 23,08 & 22,1 \\
\hline $\begin{array}{c}\text { App } \\
5\end{array}$ & 20 & 28,3 & 20 & 20,1 & 6,99 & 20 & 16,5 & 16,5 & 23,08 & 19,5 \\
\hline
\end{tabular}
característica, el procedimiento algebraico es el mismo con lo cual se entrega en la Tabla 5 los resultados de dichos análisis.

Tabla 5. Resultados Luego de Aplicar AHP

Se puede ver en la Tabla 5 que la aplicación 2 es la mejor calificada con 25,05\% y el producto que según la evaluación de los expertos tiene menores características de calidad es la App 1 con un 15,29\% de calificación. Sin embargo, la evaluación del AHP califica las aplicaciones sin tener en cuenta cuando estos productos son aptos para el uso sin poner en peligro la salud de las personas. Para realizar esta prueba se debe hacer una evaluación por lista de cotejo.

\subsection{Aplicando Rúbrica de Evaluación}


En esta etapa se propone evaluar las aplicaciones que se presentan para determinar en qué casos existe riesgo para la salud al utilizarlas. Para tal fin se presenta una Rúbrica de Evaluación para evaluar las características deseables, en la Tabla 6 se observan las primeros cuatro atributos de calidad y las opciones de calificación según el caso. Una aplicación que no cumple el atributo de calidad obtiene una calificación de cero "0", en cambio, cuando el atributo es cumplido a cabalidad su calificación máxima será de "9". El rango de calificaciones propuesto se basa en los parámetros presentados por Thomas L. Saaty para el AHP.

\begin{tabular}{|c|c|c|c|c|c|}
\hline \multicolumn{6}{|c|}{ Rúbrica de calificación de características de calidad } \\
\hline \multirow[b]{2}{*}{$\begin{array}{l}\text { Aplicación } \\
\text { validada } \\
\text { por parte } \\
\text { de un } \\
\text { grupo de } \\
\text { especialista } \\
\text { s médicos } \\
\text { para su } \\
\text { publicación. }\end{array}$} & 0 & 3 & 5 & 7 & 9 \\
\hline & $\begin{array}{l}\text { No hay } \\
\text { evidencia de } \\
\text { validación } \\
\text { por parte de } \\
\text { expertos. }\end{array}$ & $\begin{array}{c}\text { Existe duda } \\
\text { razonable } \\
\text { sobre la } \\
\text { autenticidad } \\
\text { de la } \\
\text { validación } \\
\text { médica para } \\
\text { la } \\
\text { publicación } \\
\text { de la App. }\end{array}$ & $\begin{array}{c}\text { Esta } \\
\text { validada la } \\
\text { aplicación, } \\
\text { pero no por } \\
\text { expertos } \\
\text { del área. }\end{array}$ & $\begin{array}{l}\text { Aplicación } \\
\text { validada } \\
\text { por } \\
\text { expertos } \\
\text { médicos de } \\
\text { otro país. }\end{array}$ & $\begin{array}{l}\text { Aplicación } \\
\text { validada } \\
\text { por } \\
\text { expertos } \\
\text { médicos en } \\
\text { el país de } \\
\text { publicación. }\end{array}$ \\
\hline $\begin{array}{c}\text { Documenta } \\
\text { ción } \\
\text { completam } \\
\text { ente en } \\
\text { idioma } \\
\text { oficial. Se } \\
\text { enuncian } \\
\text { las fuentes } \\
\text { de } \\
\text { información } \\
\text { y } \\
\text { funcionami } \\
\text { ento de la } \\
\text { aplicación. }\end{array}$ & $\begin{array}{c}\text { No hay } \\
\text { documentaci } \\
\text { ón de ningún } \\
\text { tipo. }\end{array}$ & $\begin{array}{c}\text { Existen } \\
\text { dudas sobre } \\
\text { la exactitud } \\
\text { de la } \\
\text { traducción y } \\
\text { sobre las } \\
\text { fuentes de } \\
\text { información. }\end{array}$ & $\begin{array}{l}\text { Documenta } \\
\text { ción } \\
\text { parcialment } \\
\text { e en el } \\
\text { idioma } \\
\text { oficial o mal } \\
\text { traducido, } \\
\text { pero se } \\
\text { entiende la } \\
\text { documenta } \\
\text { ción sobre } \\
\text { el } \\
\text { funcionami } \\
\text { ento del } \\
\text { producto. }\end{array}$ & $\begin{array}{l}\text { Traducción } \\
\text { completa } \\
\text { en el } \\
\text { idioma } \\
\text { oficial. Se } \\
\text { presentan } \\
\text { fuentes de } \\
\text { información } \\
\text {. Sin } \\
\text { embargo, } \\
\text { no se } \\
\text { explica el } \\
\text { funcionami } \\
\text { ento del } \\
\text { producto. } \\
\end{array}$ & $\begin{array}{c}\text { Documenta } \\
\text { ción en el } \\
\text { idioma } \\
\text { oficial. } \\
\text { Fuentes de } \\
\text { información } \\
\text { actuales y } \\
\text { verídicas. } \\
\text { Funcionami } \\
\text { ento de la } \\
\text { aplicación } \\
\text { claro y } \\
\text { preciso. }\end{array}$ \\
\hline $\begin{array}{c}\text { Todas las } \\
\text { decisiones } \\
\text { y } \\
\text { diagnóstico } \\
\text { s validados } \\
\text { por } \\
\text { expertos } \\
\text { médicos. }\end{array}$ & $\begin{array}{c}\text { Toma } \\
\text { decisiones y } \\
\text { genera } \\
\text { diagnósticos } \\
\text { sin } \\
\text { validación } \\
\text { médica o no } \\
\text { toma } \\
\text { decisiones. }\end{array}$ & $\begin{array}{c}\text { El producto } \\
\text { toma } \\
\text { decisiones y } \\
\text { genera } \\
\text { diagnósticos } \\
\text { con } \\
\text { validaciones } \\
\text { de personal } \\
\text { no experto. }\end{array}$ & $\begin{array}{c}\text { Toma } \\
\text { decisiones } \\
\text { y genera } \\
\text { Diagnóstico } \\
\text { s validados } \\
\text { por } \\
\text { personal } \\
\text { médico en } \\
\text { otro idioma. }\end{array}$ & $\begin{array}{c}\text { Genera } \\
\text { Diagnóstico } \\
\text { s validado } \\
\text { por } \\
\text { personal } \\
\text { médico } \\
\text { indirectame } \\
\text { nte. }\end{array}$ & $\begin{array}{c}\text { Genera } \\
\text { diagnóstico } \\
\text { s validado } \\
\text { por } \\
\text { personal } \\
\text { médico en } \\
\text { tiempo real } \\
\text { y de forma } \\
\text { directa. }\end{array}$ \\
\hline
\end{tabular}




\begin{tabular}{|c|c|c|c|c|c|}
\hline $\begin{array}{c}\text { Solicita y } \\
\text { utiliza } \\
\text { únicamente } \\
\text { los } \\
\text { recursos } \\
\text { necesarios } \\
\text { para } \\
\text { cumplir con } \\
\text { el objetivo } \\
\text { del diseño } \\
\text { del } \\
\text { producto. }\end{array}$ & $\begin{array}{c}\text { Solicita y/o } \\
\text { utiliza } \\
\text { recursos del } \\
\text { dispositivo } \\
\text { móvil más } \\
\text { allá de las } \\
\text { necesidades } \\
\text { básicas de la } \\
\text { App, accede } \\
\text { a } \\
\text { información } \\
\text { privada del } \\
\text { usuario. }\end{array}$ & $\begin{array}{c}\text { Usa recursos } \\
\text { más allá de } \\
\text { las } \\
\text { necesidades } \\
\text { de la App, } \\
\text { pero no } \\
\text { accede a la } \\
\text { información } \\
\text { privada del } \\
\text { usuario. }\end{array}$ & $\begin{array}{c}\text { Solicita } \\
\text { recursos } \\
\text { adicionales } \\
\text { al objetivo } \\
\text { de } \\
\text { funcionami } \\
\text { ento de la } \\
\text { App, pero } \\
\text { el usuario } \\
\text { puede } \\
\text { negarse al } \\
\text { acceso y } \\
\text { usar la } \\
\text { aplicación. }\end{array}$ & $\begin{array}{c}\text { No solicita } \\
\text { ni utiliza } \\
\text { recursos } \\
\text { adicionales } \\
\text { al objetivo } \\
\text { de } \\
\text { funcionami } \\
\text { ento de la } \\
\text { App, pero } \\
\text { podría } \\
\text { poner en } \\
\text { peligro la } \\
\text { privacidad } \\
\text { de los } \\
\text { datos del } \\
\text { usuario. }\end{array}$ & $\begin{array}{c}\text { Utiliza } \\
\text { recursos } \\
\text { necesarios } \\
\text { para } \\
\text { mantener } \\
\text { calidad } \\
\text { funcional } \\
\text { del } \\
\text { producto, } \\
\text { se respeta } \\
\text { privacidad } \\
\text { del usuario. }\end{array}$ \\
\hline
\end{tabular}

Tabla 6. Rúbrica de Evaluación para Características de Calidad en app (ISO, 2013, 2015 y 2016)

\section{RESULTADOS}

Una vez registradas las calificaciones se realiza una revisión de valores en la Tabla 7 para cada columna utilizando como referencia las condiciones de la Tabla 8 se asigna la calificación obtenida. Las condiciones para cada columna indican cuando una App tiene la mitad de las características con calificaciones de 7 o 9 entonces su calificación corresponderá a 5 . Cuando una app tiene el $80 \%$ de las calificaciones con 7 o 9 su calificación será de 7 , cuando la app cuenta con el total de las características de calidad con calificación de 7 o 9 tendrá una calificación de 9. El resultado del análisis para cada columna se registra en la Tabla 7 en la parte de "calificación obtenida".

\begin{tabular}{|c|c|c|c|c|c|}
\hline Características & APP & APP & APP & APP & APP \\
(elementos de calidad) & $\mathbf{1}$ & $\mathbf{2}$ & $\mathbf{3}$ & $\mathbf{4}$ & $\mathbf{5}$ \\
\hline a & 0 & 0 & 0 & 0 & 0 \\
\hline b & 3 & 7 & 9 & 9 & 9 \\
\hline c & 0 & 0 & 0 & 0 & 0 \\
\hline d & 3 & 9 & 5 & 7 & 7 \\
\hline e & 3 & 9 & 3 & 5 & 3 \\
\hline f & 0 & 0 & 0 & 0 & 0 \\
\hline g & 3 & 3 & 0 & 5 & 3 \\
\hline h & 0 & 5 & 3 & 3 & 3 \\
\hline i & 5 & 9 & 9 & 9 & 9 \\
\hline Calificación obtenida & 0 & 0 & 0 & 0 & 0 \\
\hline Usar tabla de validación para reportar calificaciones \\
\hline \multicolumn{5}{|l}{} \\
\hline \multicolumn{5}{|l}{}
\end{tabular}




\section{Tabla 7. Calificaciones de APP}

La calificación por lista de cotejo para las aplicaciones móviles sirve para detectar a partir de una validación de calidad funcional cuales productos son confiables y pueden ser usados sin temer un impacto en la salud.

\begin{tabular}{|c|c|}
\hline \multicolumn{2}{|c|}{ Validación de la app } \\
\hline $\begin{array}{c}\text { Si la aplicación posee } \\
\text { Menos de la mitad de las } \\
\text { características con 7 o 9 }\end{array}$ & 0 \\
\hline $\begin{array}{c}\text { La mitad de las características } \\
\text { con } 7 \text { o } 9\end{array}$ & 5 \\
\hline $\begin{array}{c}\text { 8 de cada } 10 \text { características } \\
\text { con 7 o 9 }\end{array}$ & 7 \\
\hline $\begin{array}{c}\text { todas las características tienen } \\
7 \text { o } 9\end{array}$ & 9 \\
\hline
\end{tabular}

Tabla 8. Métrica de Evaluación para Lista de Cotejo

Según el resultado mostrado en la Tabla 7, ninguna de las cinco aplicaciones analizadas tiene una calificación aprobatoria, lo cual significa que ninguna es una aplicación confiable, puesto que no cumplen con los atributos de calidad de la norma ISO/IEC 9126 en los atributos de calidad muestra utilizados para la evaluación de las aplicaciones.

\section{CONCLUSIONES}

Combinar el proceso analítico jerárquico con una escala de calificación permite determinar si las características de un producto software cumplen con una regulación determinada, no necesariamente aplicado a productos médicos, pero si aplicado a productos regulatorios. Aun así, puede utilizarse para tener un valor duro de calificación sobre algún aspecto determinado, por ejemplo, utilizando como experto evaluador a los clientes, usuarios o administradores del sistema, permitiendo que estos evalúen y extraigan una calificación sobre el producto que les presentan. En definitiva, lo que se busca es tener una forma de evaluar software sin tener que usar métodos subjetivos convirtiendo valoraciones cualitativas en cuantitativas. 


\section{REFERENCIAS}

Fernandez, S., Ramos, J. (2014). New technologies, apps and their application in evidence-based clinical practice. Enferm Clin núm 24. doi: https://doi.org/10.1016/j.enfcli.2014.02.001, Recuperado en septiembre de 2018. pp. 99-101

Garcia, S., Martinez, J. (2016). Apps de Salud: Nuevas Herramientas para el cuidado del Paciente Cardiológico. Envermería en Cardiología núm 22. Pp 2834

International Organization for Standardization. (2013). ISO 15504: Determinación de la Capacidad de Mejora del Proceso de Software.Ginebra: ISO

International Organization for Standardization. (2015). ISO 15288: Ingeniería de sistemas y software. Procesos del ciclo de vida del sistema.Ginebra: ISO

International Organization for Standardization. (2016). ISO 13485: Sistemas de Gestión de la Calidad de Equipos Médicos - Guidance.Ginebra: ISO

Muñoz, C., Piattini, G., Moraga, A. (2010). Calidad del producto y proceso software. Madrid: Ra-Ma. ISBN: 8478979611,9788478979615.

Redrován, F., Loja, N., Correa, k., Piña, J. (2017). Comparación de métricas de calidad para el desarrollo de aplicaciones web. 3C Tecnología. Glosas de Innovación aplicadas a la pyme núm 7(3). http://dx.doi.org/10.17993/3ctecno.2018.v7n3e27.94-113/, Recuperado en Septiembre de 2018. pp. 94-113

Saaty, T.L. (2011). Fundamentals of Decision Making and Priority Theory with AHP. RWS Publications, Pittsburg. ISBN: 0962031763,978-0962031762

Tuya, J., Ramos, I., Dolado, J. (2007). Técnicas cuantitativas para la gestión de ingeniería de software. Oleiros: Netbiblo. ISBN:9788497452045.

U.S. Food and Drug Administration. (2016). Codigó de las Regulaciones Federales 21 parte 11: Electronic Records; Electronic signatures - Guidance. Estados Unidos: GMP publications. ISBN: 1933734035 
U.S. Food and Drug Administration. (2016). Codigó de las Regulaciones Federales 21 parte 820: Quality System Regulation- Guidance. Estados Unidos: GMP publications. ISBN: 1933734507

Venegas, J., (2019). Modelo de proceso de desarrollo de software MW para productos regulados. Tesis de doctorado, Universidad Juarez Autónoma de Tabasco, México.

\section{NOTAS BIOGRÁFICAS}

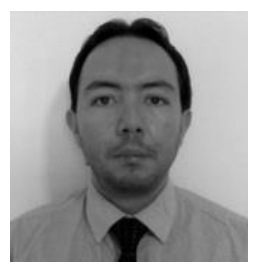

M.C.I. Jonathan Venegas Sandoval Estudiante de Doctorado en Ciencias de la Computación por la Universidad Juárez Autónoma de Tabasco, Maestro en Ciencias de Ingeniería (2014) por la Universidad Politécnica de Aguascalientes. Ingeniero Industrial (2008) Universidad Autónoma de Colombia.

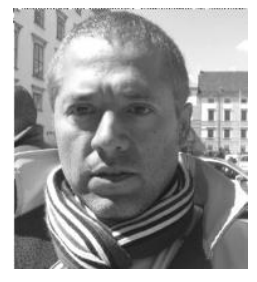

Dr. Francisco Javier Álvarez Rodríguez, Profesor de Ingeniería de Software adscrito al Departamento de Ciencias de la Computación, Universidad Autónoma de Aguascalientes (U.A.A.). Doctor en Metodología de la Enseñanza por el IMEP (México). Doctor en Ingeniería por la UNAM (México). Ha sido Decano del Centro de Ciencias Básicas en la U.A.A., así como Jefe de Departamento de Sistemas Electrónicos. Miembro de núcleos académicos de diversos posgrados de la U.A.A.

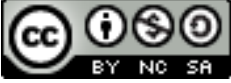

Esta obra está bajo una licencia de Creative Commons Reconocimiento-NoComercial-Compartirlgual 2.5 México. 